\title{
Outcomes of Adult Patients with Small Body Size Supported with a Continuous-Flow Left Ventricular Assist Device
}

\author{
Sanguin Lee, * Jason N. Katz,† Ulrich P. Jorde, † Nader Moazami, § Ranjit John, 9 Kartik S. Sundareswaran, II \\ David J. Farrar, || and O. H. Frazier\#
}

\begin{abstract}
There is insufficient data on patients with small body size to determine if this should be considered a risk factor for continuous-flow left ventricular assist device (CF-LVAD) support. We sought to evaluate survival outcomes, adverse events, and functional status of CF-LVAD patients with body surface area (BSA) $<1.5 \mathrm{~m}^{2}$ in a large national registry. Adults with BSA $<1.5$ $\mathbf{m}^{2}(\mathbf{n}=128)$ implanted with a HeartMate II (HMII)-LVAD from the Interagency Registry for Mechanically Assisted Circulatory Support registry from April 2008 to December 2012 formed this cohort. Outcomes were compared with HMII bridge to transplant (BTT) and destination therapy (DT) post approval studies. The majority of patients were female $(n=106,83 \%)$. A total of $64 \%(n=82)$ were implanted for BTT and $36 \%(n=46)$ for DT. The median BSA (range) was 1.44 (1.19-1.49) and 1.45 (1.25-1.49) $\mathrm{m}^{2}$ for BTT and DT, respectively. Overall survival 1 year post implant was $81 \% \pm 5 \%$ for BTT and $84 \% \pm 6 \%$ for DT. The most common adverse events for BTT and DT patients were bleeding (0.91, 0.88 events/patient year) and driveline infection (16\%, 0.28 events/patient year). Six months post implantation, $87 \%$ of BTT and $77 \%$ of DT patients were New York Heart Association functional class I or II. Post implant survival, functional status improvement, and adverse event profile for adult BTT and DT HMII patients with BSA $<1.5 \mathrm{~m}^{2}$
\end{abstract}

From the *Richard DeVos Heart \& Lung Transplant Program, Spectrum Health, Grand Rapids, Michigan; +Center for Heart and Vascular Care, Divisions of Cardiology and Pulmonary \& Critical Care Medicine, University of North Carolina, Chapel Hill, North Carolina; ₹Department of Medicine, Division of Cardiology, Albert Einstein College of Medicine / Montefiore Medical Center, Bronx, New York; $\S$ Department of Thoracic and Cardiovascular Surgery and the Department of Biomedical Engineering, Cleveland Clinic, Cleveland, Ohio; I Division of Cardiothoracic Surgery, University of Minnesota, Minneapolis, Minnesota; ||Research and Scientific Affairs, St. Jude Medical, St. Paul, Minnesota; and \#Center for Cardiac Support, Cardiovascular Surgery Research, Cullen Cardiovascular Research Laboratories, Texas Heart Institute, Houston, Texas.

Submitted for consideration November 2015; accepted for publication in revised form August 2016.

Disclosures: The following are all conflicts of interest and funding sources, if any, for each co-author: S.L.: Travel grant, Thoratec; J.N.K.: Travel grant, Thoratec; U.P.J.: Consultant Thoratec/HeartWare $(<\$ 5000$ annually); R.J.: Research Grant Thoratec/HeartWare; K.S.S.: Employee of Thoratec; D.J.F.: Employee of Thoratec; Stockholder of Thoratec.

Correspondence: Sangjin Lee, Richard DeVos Heart \& Lung Transplant Program, Spectrum Health Hospitals, Clinical Assistant Professor of Medicine, Michigan State University, 330 Barclay St NE, Suite 200, Grand Rapids, MI 49503. Email: sangjin.lee@spectrumhealth.org.

Copyright (C) 2016 by the ASAIO. This is an open-access article distributed under the terms of the Creative Commons Attribution-Non Commercial-No Derivatives License 4.0 (CCBY-NC-ND), where it is permissible to download and share the work provided it is properly cited. The work cannot be changed in any way or used commercially without permission from the journal.

DOI: 10.1097/MAT.0000000000000430 are favorable and comparable with outcomes published in the overall patient population. ASAIO Journal 2016; 62:646-651.

Key Words: LVAD, BSA, BMI, INTERMACS, HeartMate II

The landscape in the treatment of advanced heart failure is evolving rapidly with the increased use of continuous-flow left ventricular assist devices (CF-LVADs) as a bridge to transplant (BTT) or as destination therapy (DT). In the latest Interagency Registry for Mechanically Assisted Circulatory Support (INTERMACS) 7th annual report, there have been more than 15,000 durable Food and Drug Administration (FDA)-approved devices implanted since June 2006. ${ }^{1}$ The International Society of Heart and Lung Transplantation reports that the percentage of patients bridged with a LVAD at the time of heart transplantation has increased from $<20 \%$ in 2000 to more than $35 \%$ in $2011 .{ }^{2}$ Moreover, there is increased utilization of these devices as long-term permanent therapy or DT for advanced heart failure now accounting for up to $40 \%$ of all indications for CF-LVAD implantation in recent years. ${ }^{3}$ Outcomes in recent post FDA approval studies have been excellent, with $85 \%$ survival of those remaining on CF-LVAD support 1 year post implant for BTT patients and 74\% for DT patients. ${ }^{4,5}$

Compared with the earlier technology of pulsatile displacement pumps, CF-LVADs are smaller in size and can be implanted in the more restrictive anatomy of small patients. However, there is a paucity of data on the use and safety of CF-LVADs in adult patients with a small body surface area (BSA) $<1.5 \mathrm{~m}^{2}$. The HeartMate II clinical trial included a small body size cohort of 10 patients which indicated similar outcomes in patients with BSA $<1.5 \mathrm{~m}^{2}$ and those more than $1.5 \mathrm{~m}^{2}{ }^{6}$ To further address and clarify the use of CF-LVADs in adult patients with a small BSA in the post approval era, we sought to evaluate the survival outcomes, adverse events, and functional status of patients with BSA $<1.5 \mathrm{~m}^{2}$ implanted with a CF-LVAD in a large national registry.

\section{Methods}

\section{Patients}

The INTERMACS registry was queried to identify all adult patients $\geq 18$ years of age with a BSA $<1.5 \mathrm{~m}^{2}$ who were implanted with the HeartMate II CF-LVAD (HMII, Thoratec Corporation, Pleasanton, CA) between April 2008 and December $2012(n=128)$ for BTT $(n=82)$ or DT $(n=46)$ indications. Outcomes were compared with the HMII BTT (April to August 2008; $\mathrm{n}=169$ ) and DT (January to September 2010; $n=247$ ) post approval studies (PASs).

\section{Data Collection}

This was a retrospective registry study using the INTERMACS database. Data entry into the INTERMACS database 
Table 1. Baseline Characteristics: Small BSA Cohort

\begin{tabular}{lcc}
\hline Characteristic & BTT $(\mathrm{n}=82)$ & DT $(\mathrm{n}=46)$ \\
\hline Age (year) & & \\
$\quad<40$ & $20(24 \%)$ & $2(4 \%)$ \\
$\quad 40-59$ & $29(35 \%)$ & $9(19 \%)$ \\
$\geq 60$ & $33(40 \%)$ & $35(76 \%)$ \\
Female sex (\%) & $66(80 \%)$ & $40(87 \%)$ \\
Race & & \\
$\quad$ White & $51(62 \%)$ & $30(65 \%)$ \\
$\quad$ Black & $15(18 \%)$ & $1(2 \%)$ \\
Asian/American Indian & $6(7 \%)$ & $9(20 \%)$ \\
Unknown/other & $10(13 \%)$ & $6(13 \%)$ \\
INTERMACS profiles & $18(22 \%)$ & $5(11 \%)$ \\
$\quad$ Profile 1 & $52(64 \%)$ & $29(63 \%)$ \\
Profile 2-3 & $10(12 \%)$ & $12(26 \%)$ \\
$\quad$ Profile 4-7 & $26(32 \%)$ & $20(43 \%)$ \\
Ischemic etiology (\%) & $62(76 \%)$ & $39(85 \%)$ \\
NYHA class IV (\%) & $1.44[1.19-1.49]$ & $1.45[1.25-1.49]$ \\
Body surface area (m $\left.{ }^{2}\right)$ (median & \\
$\quad$ range]) & & \\
Weight (kg) & $47[35-53]$ & $49[39-54]$ \\
\hline
\end{tabular}

BSA, body surface area; INTERMACS, Interagency Registry for Mechanically Assisted Circulatory Support; NYHA, New York Heart Association.

is voluntary and is specified by each participating center. The adverse events definitions and variables analyzed may be accessed via the INTERMACS website. ${ }^{7}$

\section{Statistical Analysis}

Continuous variables are presented as mean \pm SD or median (range). Categorical variables are presented as proportions. Survival, adverse events, and quality of life were compared with the HMII BTT ${ }^{4}$ and $\mathrm{DT}^{5}$ PAS. Survival was evaluated using the Kaplan-Meier method. Comparison of survival between two groups was performed using the log-rank test. Adverse events are presented as both percentages and event rates (events per patient year of support; eppy). Comparisons of adverse event rates were performed using Cochran-Mantel-Haenszel statistics. All statistical comparisons were two sided with a significance level at $p<$ 0.05. Statistical analyses were performed with SigmaPlot (Cranes Software, Chicago, IL) and SAS (SAS Institute, Inc., Cary, NC).

\section{Results}

\section{Baseline Characteristics}

Table 1 shows the baseline characteristics of the small BSA $<1.5 \mathrm{~m}^{2}$ cohort stratified by BTT and DT. The most striking demographic characteristic unique to this patient population is that the majority of the patients ( $80 \%$ BTT and $87 \%$ DT) were female. The majority of patients were implanted as INTERMACS profile $2-3(64 \%$ BTT and $63 \%$ DT) and New York Heart Association (NYHA) Class IV (76\% BTT and 85\% DT). The median (range) BSA was $1.44 \mathrm{~m}^{2}(1.19-1.49)$ and $1.45 \mathrm{~m}^{2}(1.25-1.49)$ for BTT and DT patients, respectively. Age was greater in the DT group with $76 \%$ at least 60 years old compared with $40 \%$ for BTT. Tables 2 and 3 show the baseline characteristics of the HMII BTT and DT PAS cohorts, respectively. Females made up $22 \%$ and $17 \%$ of the BTT and DT PAS cohorts, respectively. The median BSA was $2.03 \pm 0.25$ and $2.01 \pm 0.29 \mathrm{~m}^{2}$ for the BTT and DT PAS cohorts, respectively.

\section{Outcomes}

Survival. The survival of the small BSA cohort was compared with the survival of the HMII BTT ${ }^{4}$ and DT ${ }^{5}$ PASs (Figures 1 and 2). For the BTT population, the 6 month and 1 year post implant survival of the small BSA cohort was $84 \% \pm 4 \%$ and $81 \%$ $\pm 5 \%$, respectively, which were similar to and not statistically different from the 6 month and 1 year post implant survival in the post approval BTT population $(90 \% \pm 2 \%$ and $84 \% \pm 3 \%$, respectively; $p=0.367$ ). For the DT population, the 1 and 2 year post implant survival of the small BSA cohort was $84 \% \pm$

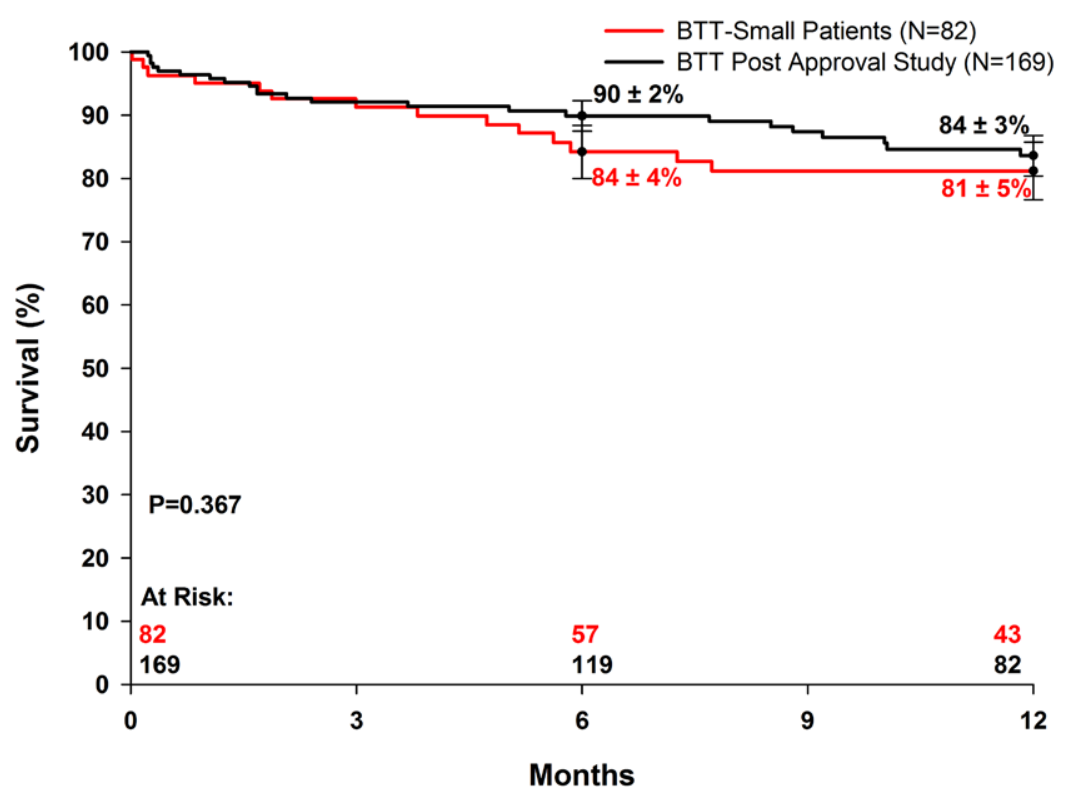

Figure 1. Kaplan-Meier survival for small BSA BTT patients compared to post approval BTT study. BSA, body surface area; BTT, bridge to transplant. $\frac{\text { full color }}{\text { online }}$ 


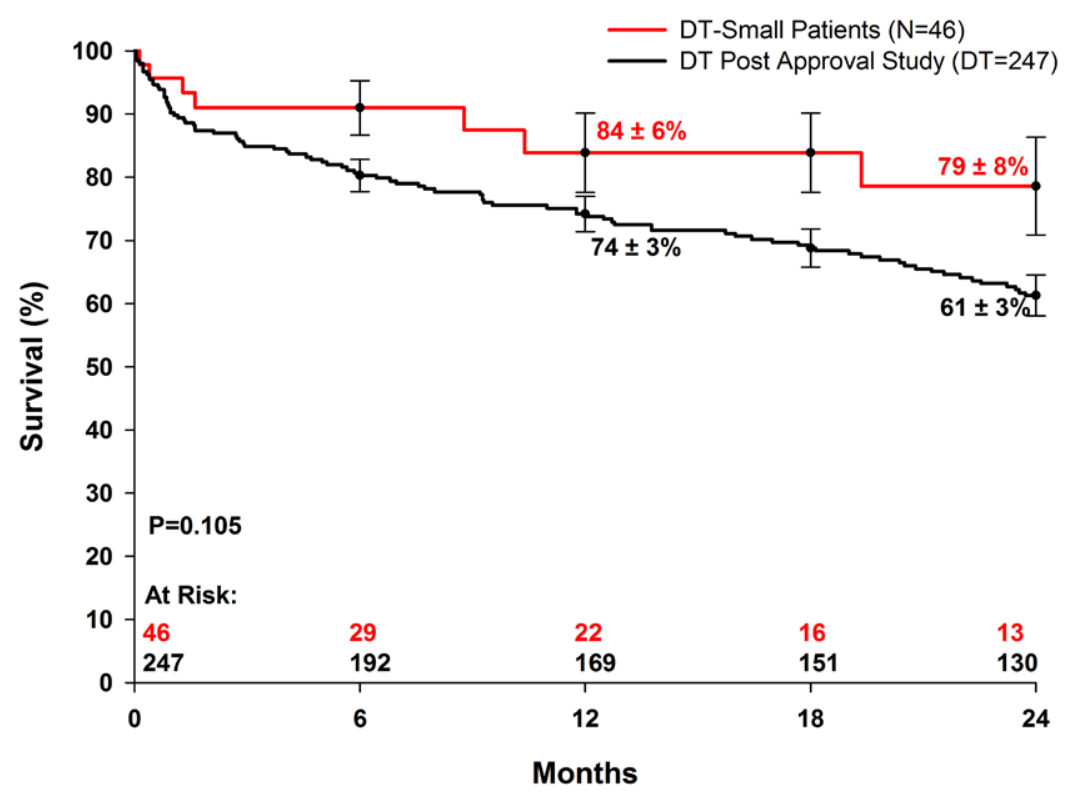

Figure 2. Kaplan-Meier survival for small BSA DT patients compared to postapproval DT study. BSA, body surface area; DT, destination therapy. [utulcolor

$6 \%$ and $79 \% \pm 8 \%$, respectively, and trended higher but was not statistically different from the 1 and 2 year post implant survival in the post approval DT population $(74 \% \pm 3 \%$ and $61 \% \pm 3 \%$, respectively; $p=0.105$ ).

Adverse Events. The adverse event rates for the small BSA cohort are similar to the BTT and DT post approval patients (Tables 4 and 5, respectively). However, in the BTT population, bleeding was significantly lower in the small BSA cohort $(0.91$ vs. 1.44 eppy; $p=0.010$ ). In DT patients, hemolysis occurred more frequently in the small BSA patients $(0.16 \mathrm{vs} .0 .06$ eppy; $p=0.014)$.

Functional Status. Both BTT and DT small BSA patients had a marked improvement in NYHA Classification. Before implant, no patients reported NYHA Class I/II symptoms. At
6 months post implant, more than $75 \%$ of small BSA patients had improved to NYHA class $1 /$ II with sustained improvement through 12 months (Figure 3A, B).

\section{Discussion}

This study represents the largest cohort of adult patients with a BSA $<1.5 \mathrm{~m}^{2}$ implanted with a CF-LVAD and support the use of CF-LVAD therapy in this patient population.

The post implant survival of small BSA patients are comparable with the published HMII post approval BTT and DT studies where the mean BSA was $2.03 \pm 0.25$ and $2.01 \pm 0.29$ $\mathrm{m}^{2}$, respectively. ${ }^{4,5}$ The post implant survival of the small BSA BTT cohort was $84 \% \pm 4 \%$ and $81 \% \pm 5 \%$ at 6 months and
A

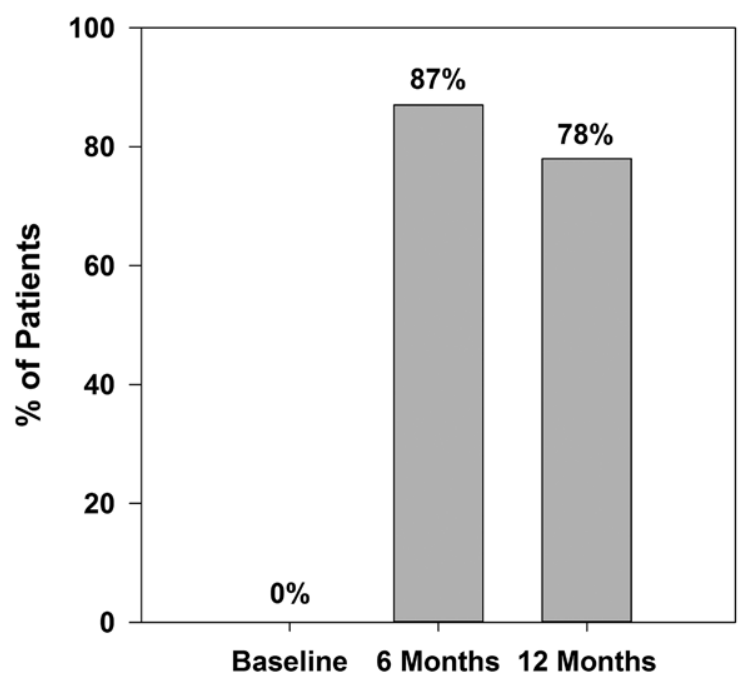

B

DT NYHA Class I or II (\%)

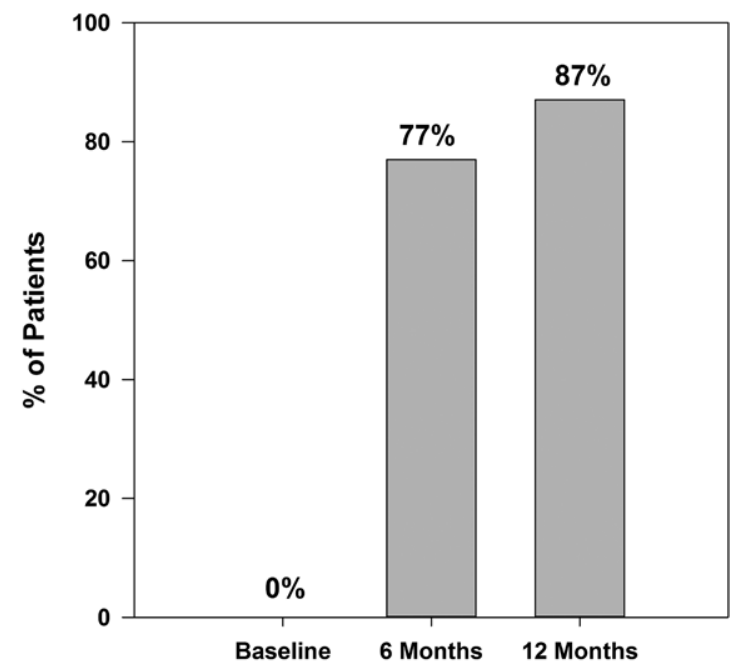

Figure 3. A: NYHA classification of BTT patients pre- and post-LVAD implant. B: NYHA classification of DT patients pre and post LVAD implant. BTT, bridge to transplant; DT, destination therapy; LVAD, left ventricular assist device; NYHA, New York Heart Association. 
Table 2. Baseline Characteristics: HMII BTT PAS Cohort

\begin{tabular}{lc}
\hline Characteristic & BTT $(\mathrm{n}=169)$ \\
\hline Age (year) & \\
$\quad<40$ & $26(15 \%)$ \\
$\quad 40-59$ & $81(48 \%)$ \\
$\quad \geq 60$ & $59(35 \%)$ \\
Female sex (\%) & $38(22 \%)$ \\
Race & \\
$\quad$ White & $125(74 \%)$ \\
$\quad$ Black & $29(17 \%)$ \\
$\quad$ Unknown/other & $15(9 \%)$ \\
INTERMACS profiles & \\
$\quad$ Profile 1 & $41(24 \%)$ \\
$\quad$ Profile 2-3 & $96(57 \%)$ \\
Profile 4-7 & $32(19 \%)$ \\
Body surface area $\left(\mathrm{m}^{2}\right)$ (median \pm SD) & $2.03 \pm 0.25$ \\
\hline
\end{tabular}

BTT, bridge to transplant; HMII, HeartMate II; INTERMACS, Interagency Registry for Mechanically Assisted Circulatory Support; PAS, post approval study.

1 year, respectively. Although not statistically significant, the small BSA DT cohort had improved 1 and 2 year post implant survival compared with the DT PAS $(84 \% \pm 6 \%$ vs. $74 \% \pm 3 \%$ and $79 \% \pm 8 \%$ vs. $61 \% \pm 3 \%$, respectively; $p=0.105)$.

The adverse event profile of the small BSA patients is equally as comparable with the post approval HMII BTT and DT studies (Tables 4 and 5). For the BTT population, the small BSA cohort had adverse event rates that were not statistically different from the HMII BTT PAS for all the major adverse events rates studied, with the exception that there was less bleeding noted in the small BSA cohort (0.91 vs. 1.44 eppy). Similarly, the adverse event profile for the DT population was not dissimilar from the post approval DT cohort, although more hemolysis was noted in the small BSA cohort $(0.16 \mathrm{vs}$. 0.06 eppy). The reason for a higher incidence of hemolysis in the small BSA cohort is unclear. Increased hemolysis may be associated with the cannula position in a small BSA patient, smaller relative circulating blood volume passing through the rotor, or other factors all of which require further investigation. However it does not appear to be related to thrombus, which was similar at 0.03 to 0.04 eppy between cohorts, and stroke at 0.04 eppy was actually trending to be lower in the smaller patients but this did not reach statistical significance.
Table 3. Baseline Characteristics: HMII DT PAS Cohort

\begin{tabular}{lc}
\hline Characteristic & DT $(\mathrm{n}=247)$ \\
\hline Age (years) & \\
$\quad<40$ & $6(2 \%)$ \\
$40-59$ & $69(28 \%)$ \\
$\geq 60$ & $172(70 \%)$ \\
Female sex (\%) & $43(17 \%)$ \\
Race & \\
$\quad$ White & $185(75 \%)$ \\
Black & $45(18 \%)$ \\
Unknown/other & $17(7 \%)$ \\
INTERMACS profiles & \\
$\quad$ Profile 1 & $18(22 \%)$ \\
Profile 2-3 & $52(64 \%)$ \\
Profile 4-7 & $10(12 \%)$ \\
Ischemic etiology (\%) & $140(57 \%)$ \\
NYHA class IV (\%) & $196(79 \%)$ \\
Body surface area (m $\left.{ }^{2}\right)($ median \pm SD) & $2.01 \pm 0.29$ \\
Weight (kg) (median \pm SD) & $85 \pm 22$ \\
\hline
\end{tabular}

DT, destination therapy; HMII, HeartMate II; INTERMACS, Interagency Registry for Mechanically Assisted Circulatory Support; NYHA, New York Heart Association; PAS, post approval study.

Paralleling comparable post implant survival outcomes and adverse event profiles of the small BSA patients is the marked improvement in NYHA functional class with the majority of patients reporting class I/II post implant, suggesting that the small BSA patients enjoy not only morbidity and mortality benefits but also improved quality of life with LVAD therapy.

The US clinical trial of the HM II also studied two small BSA patient (BSA < 1.5) cohorts, one for DT $(n=24)$ and one for BTT $(n=10)$, which were analyzed separately in the trial. The BTT cohort was composed of 10 females and was published in the device labeling. ${ }^{6}$ All small BSA patients survived to 180 days post implant with $86 \%$ improving from NYHA class IV to class I/II after 3 months. Six minute walk distance also increased an average of $230 \mathrm{~m}$ in the small BSA cohort versus $247 \mathrm{~m}$ in the primary study cohort. Adverse event rates were similar to those measured in the primary study cohort. Patients with small body size were comparable with larger patients in the clinical study; however, the number of small BSA patients was too few to make definitive conclusions.

A recent report by Cabrera et al. $^{8}$ utilizing the INTERMACS registry compared outcomes of 28 pediatric patients aged 11

Table 4. Adverse Events: BTT

\begin{tabular}{|c|c|c|c|c|c|}
\hline & \multicolumn{2}{|c|}{$\begin{array}{l}\text { HMII Patients With a Small BSA }(\mathrm{n}=82) \\
\text { (Total Duration: } 107.9 \text { Patient Years) }\end{array}$} & \multicolumn{2}{|c|}{$\begin{array}{l}\text { HMII BTT Post Approval Study }(\mathrm{n}=169) \\
\text { (Total Duration: } 142 \text { Patient Years) }\end{array}$} & \multirow[t]{2}{*}{$p$} \\
\hline & $\%$ Patients & $\begin{array}{c}\text { Events (Events/Patient } \\
\text { Year) }\end{array}$ & $\%$ Patients & $\begin{array}{c}\text { Events (Events/Patient } \\
\text { Year) }\end{array}$ & \\
\hline Bleeding & $35(43 \%)$ & $98(0.91)$ & $75(44 \%)$ & $204(1.44)$ & 0.010 \\
\hline Explant due to device thrombosis & $1(1 \%)$ & $1(0.01)$ & NR & NR & NR \\
\hline Hemolysis & $5(6 \%)$ & $6(0.06)$ & $5(3 \%)$ & $5(0.04)$ & 0.458 \\
\hline Hepatic dysfunction & $8(10 \%)$ & $8(0.07)$ & $11(7 \%)$ & $12(0.08)$ & 0.783 \\
\hline Any infection & $33(40 \%)$ & $112(1.04)$ & $78(46 \%)$ & $142(1.00)$ & 0.836 \\
\hline Drive line infection & $13(16 \%)$ & $30(0.28)$ & $30(18 \%)$ & $45(0.32)$ & 0.626 \\
\hline Pump pocket infection & $1(1 \%)$ & $1(0.01)$ & $3(1.8 \%)$ & $3(0.02)$ & 0.467 \\
\hline Stroke & 7 (9\%) & $7(0.06)$ & $11(7 \%)$ & $11(0.08)$ & 0.723 \\
\hline Renal dysfunction & $11(13 \%)$ & $11(0.10)$ & $17(10 \%)$ & $19(0.13)$ & 0.496 \\
\hline Right heart failure & $9(11 \%)$ & $12(0.11)$ & $25(15 \%)$ & $26(0.18)$ & 0.177 \\
\hline
\end{tabular}

BSA, body surface area; BTT, bridge to transplant. 
Table 5. Adverse Events: DT

\begin{tabular}{|c|c|c|c|c|c|}
\hline & \multicolumn{2}{|c|}{$\begin{array}{l}\text { HMII Patients With a Small BSA }(n=46) \\
\text { (Total Duration: 55.9 Patient Years) }\end{array}$} & \multicolumn{2}{|c|}{$\begin{array}{l}\text { HMII DT Post Approval Study }(\mathrm{n}=247) \\
\text { (Total Duration: } 386 \text { Patient Years) }\end{array}$} & \multirow[t]{2}{*}{$p$} \\
\hline & $\%$ Patients & $\begin{array}{c}\text { Events (Events/Patient } \\
\text { Year) }\end{array}$ & $\%$ Patients & $\begin{array}{c}\text { Events (Events/Patient } \\
\text { Year) }\end{array}$ & \\
\hline Bleeding & $29(43 \%)$ & $49(0.88)$ & $133(54 \%)$ & $324(0.84)$ & 0.836 \\
\hline Explant due to device thrombosis & $2(4 \%)$ & $2(0.04)$ & $10(4 \%)$ & $12(0.03)$ & 0.857 \\
\hline Hemolysis & $9(20 \%)$ & $9(0.16)$ & $16(7 \%)$ & $23(0.06)$ & 0.014 \\
\hline Hepatic dysfunction & $4(9 \%)$ & $5(0.09)$ & NR & NR & NR \\
\hline Any infection & $16(35 \%)$ & $47(0.84)$ & NR & NR & NR \\
\hline Drive line infection & $6(13 \%)$ & $11(0.20)$ & $47(19 \%)$ & $85(0.22)$ & 0.749 \\
\hline Pump pocket infection & $1(2 \%)$ & $1(0.02)$ & NR & NR & NR \\
\hline Stroke & $2(4 \%)$ & $2(0.04)$ & $29(12 \%)$ & $32(0.08)$ & 0.245 \\
\hline Renal dysfunction & $3(7 \%)$ & $3(0.05)$ & $44(18 \%)$ & $58(0.15)$ & 0.079 \\
\hline Right heart failure & $5(11 \%)$ & $5(0.09)$ & $44(18 \%)$ & $62(0.16)$ & 0.223 \\
\hline
\end{tabular}

BSA, body surface area; DT, destination therapy.

to 18 years compared with young adults aged 19 to 39 years implanted with the HMII. At 6 months, the composite of survival to transplantation, ongoing support, or recovery was $96 \%$ for the pediatric group, not dissimilar to the young adult group $(p=0.330)$. The median BSA for the pediatric group was 1.91 $\mathrm{m}^{2}(1.47-2.65)$.

Interestingly, the results of the Japanese HMII prospective BTT trial were recently published..$^{9}$ The six patients enrolled in this study had a mean BSA of $1.58 \pm 0.17 \mathrm{~m}^{2}$. All patients were inotrope dependent and failing medical management at baseline. Although a small trial, at 6 months post implant, there were no deaths and all patients were alive with ongoing support. Functional status assessed by the 6 minute walk distance increased from $268 \pm 92 \mathrm{~m}$ at baseline to $399 \pm 105 \mathrm{~m}$, and $100 \%$ of the patients were reported to be NYHA class I/II in follow-up. All the six patients eventually were successfully transplanted.

Unlike previous studies, but similar to the small patient cohort in the US clinical trial, this report is unique in that the study population is predominantly female. The majority of clinical trials and investigations in heart failure have traditionally been comprised of predominantly male populations. In the mechanical circulatory support literature, the female sex is particularly underrepresented. This is especially true historically with the use of the first-generation pulsatile displacement pumps, where smaller anatomies were physically unable to accommodate the larger devices. A recent single center study looked at sex-specific outcomes in the CF-LVADs era. ${ }^{10}$ Their study population included 130 patients, 35 (27\%) of whom were female. The investigators found comparable short and mid-term post implant survival outcomes, hospital length of stay, readmissions rates, and post operative complications between males and females.

Boyle et al. ${ }^{11}$ retrospectively analyzed the pre operative risks for bleeding and stroke during CV-LVAD support in the HMII BTT and DT clinical trials. Of 956 patients, $220(23 \%)$ were female. The authors found an increased incidence of thrombotic and hemorrhagic events in female patients compared with male patients. In this report, $83 \%$ of the study population was female, and with comparable post implant survival outcomes and adverse event rates matching the post approval LVAD studies, it may be suggested that females benefit as much from CF-LVAD therapy as their male counterparts.

\section{Limitations}

This study has several important limitations. The PASs included the small BSA cohort but because the majority of patients in the PAS had a BSA $>1.5 \mathrm{~m}^{2}$, the impact of this inclusion would not be clinically significant. The BTT and DT PAS cohorts were not implanted during the same time-frame which may have influenced outcomes as management strategies are expected to improve with time and experience. The INTERMACS database was designed as a registry and not for the purpose of analyzing outcomes in small BSA patients implanted with CF-LVADs. Furthermore, the survival outcomes and adverse event rates of the small BSA patients compared with the post approval BTT and DT studies were not risk adjusted.

In conclusion, this study represents the largest cohort of adult patients analyzed with a small $B S A<1.5 \mathrm{~m}^{2}$ receiving a CF-LVAD. The majority of the patient population was female. With comparable post implant survival outcomes and adverse event rates comparable with the published post approval BTT and DT studies, this study supports the use of CF-LVADs as BTT or DT in patients with a small BSA $<1.5 \mathrm{~m}^{2}$.

\section{References}

1. Kirklin JK, Naftel DC, Pagani FD, et al. Seventh INTERMACS annual report: 15,000 patients and counting. J Heart Lung Transplant 34: 1495-1504, 2015.

2. Lund LH, Edwards LB, Kucheryavaya AY, et al; International Society for Heart and Lung Transplantation: The Registry of the International Society for Heart and Lung Transplantation: Thirtieth Official Adult Heart Transplant Report-2013; focus theme: age. J Heart Lung Transplant 32: 951-964, 2013.

3. Kirklin JK, Naftel DC, Pagani FD, et al: Sixth INTERMACS annual report: A 10,000-patient database. J Heart Lung Transplant 33: 555-564, 2014.

4. Starling RC, Naka Y, Boyle AJ, et al: Results of the post-U.S. Food and Drug Administration-approval study with a continuous flow left ventricular assist device as a bridge to heart transplantation: A prospective study using the INTERMACS (Interagency Registry for Mechanically Assisted Circulatory Support). J Am Coll Cardiol 57: 1890-1898, 2011.

5. Jorde UP, Kushwaha SS, Tatooles AJ, et al; HeartMate II Clinical Investigators: Results of the destination therapy post-food and drug administration approval study with a continuous flow left ventricular assist device: A prospective study using the INTERMACS registry (Interagency Registry for Mechanically Assisted Circulatory Support). J Am Coll Cardiol 63: 1751-1757, 2014. 
6. HeartMate II Left Ventricular Assist System Instructions for Use. 106020.G ed. 2014, Pleasanton, CA: Thoratec Corporation.

7. Kirklin JK, Naftel DC, Kormos RL, et al. Fifth INTERMACS annual report: Risk factor analysis from more than 6,000 mechanical circulatory support patients. J Heart Lung Transplant 32: 141-156, 2013.

8. Cabrera AG, Sundareswaran KS, Samayoa AX, et al: Outcomes of pediatric patients supported by the HeartMate II left ventricular assist device in the United States. I Heart Lung Transplant 32: 1107-1113, 2013.

9. Kyo S, Ono M, Sawa Y, et al; HeartMate II Japanese Clinical Investigators: Results of the prospective multicenter Japanese bridge to transplant study with a continuous-flow left ventricular assist device. J Artif Organs 17: 142-148, 2014.

10. Tsiouris A, Morgan JA, Nemeh HW, Hodari A, Brewer RJ, Paone G: Sex-specific outcomes in patients receiving continuous-flow left ventricular devices as a bridge to transplantation or destination therapy. ASAIO J 60: 199-206, 2014.

11. Boyle AJ, Jorde UP, Sun B, et al; HeartMate II Clinical Investigators: Pre-operative risk factors of bleeding and stroke during left ventricular assist device support: An analysis of more than 900 HeartMate II outpatients. J Am Coll Cardiol 63: 880-888, 2014 